\title{
Assessment of line of sight characteristics of ITER bolometer prototype collimators
}

\author{
F. Penzel ${ }^{\mathrm{a}}$, H. Meister ${ }^{\mathrm{a}}$, L. Giannone ${ }^{\mathrm{a}}$, M. Kannamüller ${ }^{\mathrm{a}}$, J. Koll $^{\mathrm{a}}$, T. Trautmann ${ }^{\mathrm{a}}$, A. W. Koch ${ }^{\mathrm{b}}$ \\ ${ }^{a}$ Max-Planck-Institute for Plasmaphysics, EURATOM Association, Garching, Germany \\ ${ }^{b}$ Institute for Measurement Systems and Sensor Technology, Technical University of Munich
}

\begin{abstract}
This work outlines the present design status of developments on the optimization of line of sight (LOS) characteristics for the ITER bolometry collimators. The verification and measurement of the LOS of the bolometry is an important issue for a reliable operation of the tomographic reconstruction algorithms. Therefore the ITER Bolometer Robot Test Facility (IBOROB) is used as a diagnostic tool in order to analyse signal geometry and assess the performance of different prototypes. The LOS characteristics as a function of number of apertures is presented and the influence of a microwave filtering aperture was determined. The results of the improvement of stray light attenuation by application of the graphite based coating $\mathrm{AQUADAG}^{\circledR}$ are evaluated as well and an overview about the current collimator design is given. This paper focuses on the most remarkable results and a way to further possible upgrades is outlined. However, some results are not in accordance with the ones predicted in theoretical calculations. Thus not all key parameters which influence the LOS could be clearly identified yet.
\end{abstract}

Keywords: ITER, measurement by laser beam, bolometer, remote handling, collimator, robot

\section{Introduction}

A crucial element of the future bolometer diagnostic for ITER is the collimator. It defines the geometry and characteristics of the LOS. As the integration of the diagnostic in ITER requires the LOS to pass through narrow gaps and thus the LOS to have small opening angles, the characteristics of the envisaged design for the collimators need to be tested on prototypes.

Considering the fraction of light from the laser reaching the detector, as shown in [1], the ideal transmission function can be calculated analytically. Comparison of the measured values allows to assess the impact of stray light respectively of reflections. To achieve the aim of systematic measurements of the transmission functions of collimator prototypes, the ITER Bolometer Robot Test Facility (IBOROB) has been set up and brought into operation. A set of about 100 different collimator configurations were examined. The results achieved have helped to evaluate the different design versions and optimize the means for stray light reduction. Theoretical analysis has already indicated, that the number of apertures inside the collimator influences the stray light

Email address: florian.penzel@ipp.mpg.de (F. Penzel) from neighboring channels. The wall design, especially the surface has a major influence on the LOS by reducing or allowing for reflections.

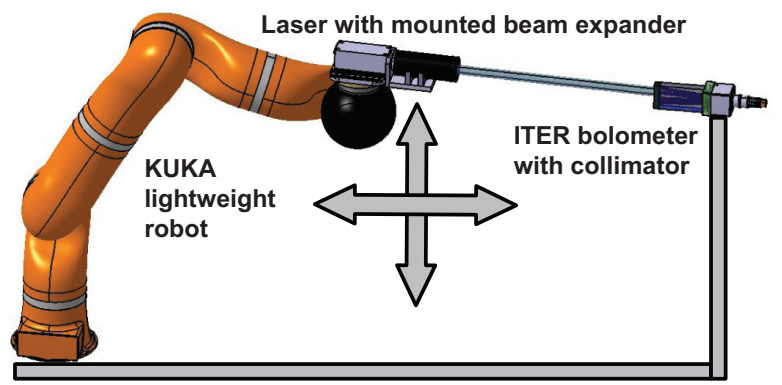

Figure 1: Schematic drawing of IBOROB: KUKA lightweight robot with laser and mounted beam expander. The bolometer is on the right.

\section{Experimental set-up}

The method used for the assessment of the LOS characteristic of the ITER bolometer diagnostic components is based on previous work performed at JET [2]. It is currently under development for the utilization as a LOS calibration device in ASDEX Upgrade, the viability of the proposed method was shown previously [1]. 
The laboratory assembly IBOROB consists of a lightweight robot from KUKA Robotics, which is used to efficiently position a laser on many points, covering the complete viewing cone of each LOS and to direct the beam precisely into the entrance aperture of the bolometer. Measuring the response of the bolometer and simultaneously the position of the robot allows for the calculation of the transmission function, the angular étendue and finally the geometric function in reconstruction space. A schematic drawing of the experiment can be seen in figure 1 .

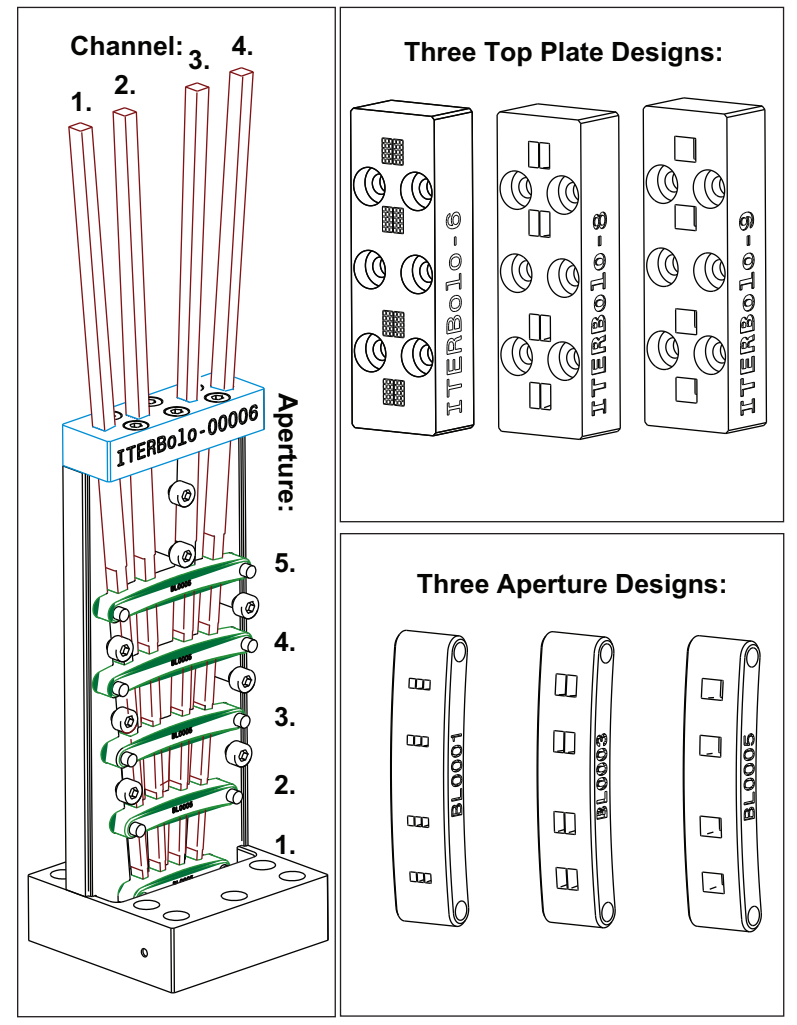

Figure 2: CAD drawing of the collimator prototype used for the measurement of the LOS on the left. Three top plate and aperture designs are shown on the right. For proper identification, the four channels and five apertures are numbered sequentially.

The facility was upgraded for performing the measurement presented in this work. It now uses an industrial single-mode diode laser (iBeam smart 640-S, Ultra-Compact Diode Laser, TOPTICA Photonics AG). The output power can be controlled up to $100 \mathrm{~mW}$.

Furthermore a beam expander (NT55-579, 20x Complete Beam Expander, Edmund Optics) was added. It was mounted in front of the laser to increase the diameter of the input beam to a larger parallel output beam. Additionally, it enables control of the beam di- ameter which is a desired feature when the collimator is mounted at a certain distance (here: $\sim 500 \mathrm{~mm}$ ) from the robot. The laser is expanded to be at least twice as big as the top plate aperture opening to generate the most uniform and constant power deposition as possible.

Several prototypes have been built using the molybdenum super-alloy TZM (titanium, zirconium, molybdenum) and aluminum. TZM is planned to be used for various ITER parts because of its superior thermomechanical properties. A CAD drawing of the basic bolometer prototype design used for the measurement of the LOS can be seen in Figure 2. It also shows different top plate and aperture designs which can be mounted on the bolometer. In total, the current version of the collimator body can be equipped with up to five apertures.

The collimator is assembled from two parts which are mounted by screws. Top plate and detector housing are also attached with screws. The collimator has four channels. Basically, the étendue of each channel is characterized by the construction parameters of the collimator like cover plate size, channel width and diameter of the integrated apertures. The analyzed collimator is otherwise identical to the old version analyzed in [1] and the channels have a similarly shaped field of view. Only its opening angle is determined by the new apertures. Some apertures were manufactured with integrated subchannels to reduce the viewing cone in toroidal direction, which vary in number and size. The top plate can be a simple rectangular opening or alternatively, be equipped with a microwave filtering grid. The grid consists of small channels which act as an attenuating waveguide for microwaves in the frequency range for ITER $(\sim 170 G h z)$. A standardized test program for the measurement was applied, with an emphasis on reliability and reproducibility. All necessary parameters for the measurement, like the coordinate systems, the start positions, the step sizes can be controlled by a LabVIEW program with the ImagingLab Robotics Library Toolkit for KUKA. It was combined with the bolometer data acquisition and implemented in LabVIEW according to [3]. For safety reasons there has been the requirement of having a man in the loop at all times. This means that during automated operation there is always an operator who is able to intervene in the preprogrammed plan in case of potential hazards.

The measurements in this work are presented in the following way: The transmission function normalized to maximum value (voltage of the Wheatstone bridge from the investigated detector channel) is plotted against the inclination angle in the poloidal (vertical) or toroidal (horizontal) angle. The angles are defined between the normal of the top plate and the laser beam. 
The absolute alignment of the facility was done manually with a measurement $\operatorname{arm}\left(\right.$ FaroArm $^{\circledR}$ Platinum $)$.

\section{Results and discussion}

The main purpose was to evaluate the various collimator configurations and design versions shown in figure 2 in order to approach the target specifications. The LOS are influenced by the characteristics of the channels, apertures, walls and reflections, the used materials and by the manufacturing process. Options such as using multiple aperture configurations with and without top plates are compared to each other, aimed to understand the signal characteristics. In parallel, a big database was established which helped to see common features of the different designs and discover future risks. The behavior of the different prototypes for a small viewing cone $\left(F W H M \sim 1^{\circ}\right)$ as a function of number of apertures is presented in figure 3 .

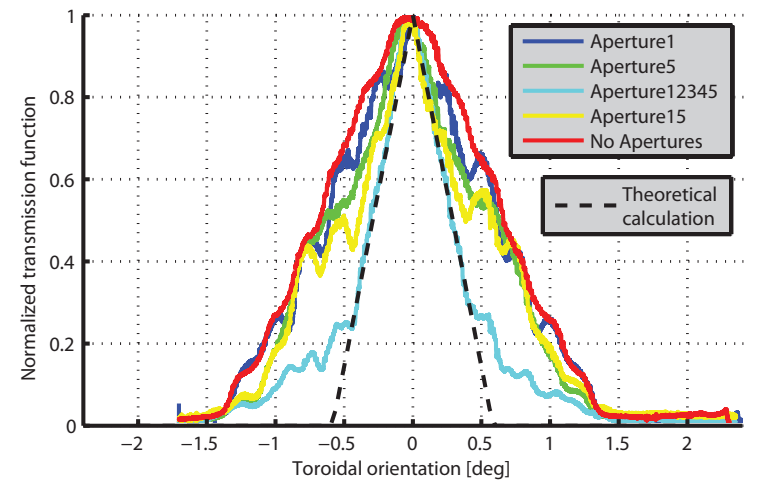

Figure 3: Comparison of five different aperture configurations. A set of all apertures (Apertures 12345), the first (Apertures1), the last (Apertures5), a combination (Apertures15) and without apertures (No Apertures) at all.

These measurements, here in the toroidal orientation, effectively determine the minimum number of apertures that are necessary for a given channel width. In comparison with the ideal signal strong stray light $\left(\left[-0.5^{\circ},-1^{\circ}\right] \&\left[0.5^{\circ}, 1^{\circ}\right]\right)$ is evident which broadens the signal. This measurement shows that for obtaining a small étendue multiple apertures are absolutely necessary. Generally, broad viewing cones $\left(F W H M \sim 3^{\circ}\right)$ show a much lesser impact of stray light because of the less abrupt signal slope as seen on figure 4 . Thus fewer apertures could be used.

Another analysis was to identify separately the influence of the microwave filtering top plate as shown in Figure 5. It shows the transmission function in poloidal

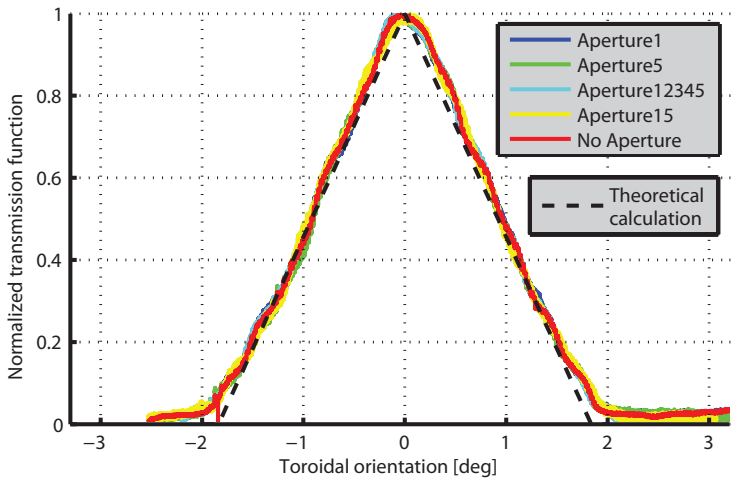

Figure 4: Comparison of the same five different aperture configurations as in figure 3 with a wider viewing cone.

orientation of measurements with (Figure 2: ITERBolo6) and without (Figure 2: ITERBolo-9) the microwave filtering grid. The collimator version with a wide viewing cone shows signal disturbances in the center. This is due to the top plate thickness of $5 \mathrm{~mm}$. Whether the grids are really required for the final bolometry design for ITER is still under discussion. However, the position of the grid could be closer to the detector so that the influence might get reduced.

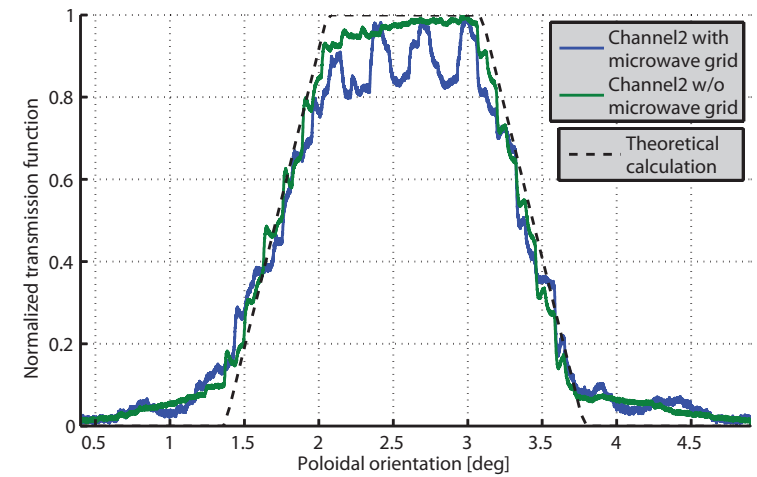

Figure 5: Comparison of two different top plate designs: with and without microwave filtering aperture.

Measurement of stray light absorption by application of a coating named AQUADAG ${ }^{\circledR 1}$ was determined as well. Because of the limited access and difficult application of ceramic coatings suitable for ITER like, e.g. $\mathrm{B}_{4} \mathrm{C}$ or $\mathrm{TiO} / \mathrm{Al}_{2} \mathrm{O}_{3}$ this coating was applied to evaluate prospects for the development of appropriate ceramic coatings. The coating is a water based colloidal graphite

\footnotetext{
${ }^{1}$ Trade name and shortened form of "Aqueous Deflocculated Acheson Graphite" (AQUADAG $\left.{ }^{\circledR}\right)$, Permanent Mold Release Coating, from Achseon Colloids Company.
} 


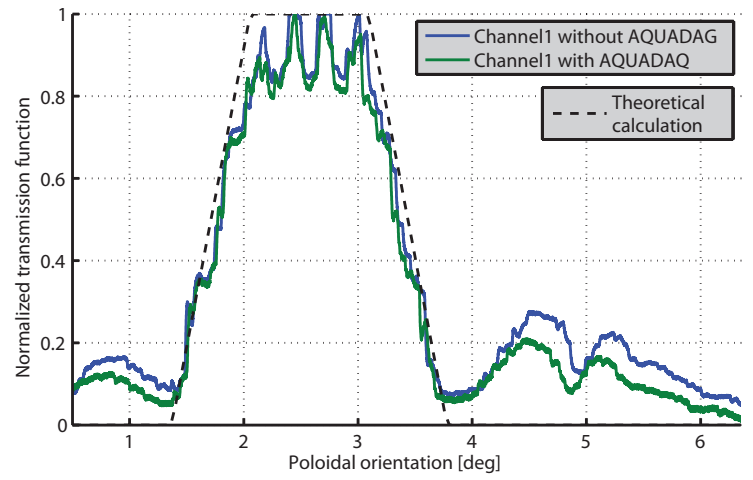

Figure 6: Analysis of reflection attenuation with and without AQUADAG ${ }^{\circledR}$ applied on the collimator body.

suspension and usually used to avoid build up of static or provide electrical screening. Due to the high content of ultra-fine particles of graphite it can also be used to enhance the radiation absorption and reduce reflections. At ASDEX Upgrade and JET bolometers it was already successfully applied.

An exact value of the difference in the absorption coefficient can not easily be given because especially at flat angles the reflection coefficient depends strongly on the surface roughness and the angle of incidence. For this analysis a collimator configuration without any apertures and a channel at the border (1) was selected. The aim was to measure the difference of the reflection absorption so a higher signal to noise ratio was expected without the apertures. Figure 6 shows the results. The radiation attenuation of the wall reflections can be seen at the poloidal orientation of $\sim 1^{\circ}$ and between $4^{\circ}$ and $6^{\circ}$. The width of the reflection corresponds approximately to the main LOS in the center. The discontinuities result from the cutout in the walls intended for the mounting of the apertures. An attenuation of roughly $30 \%$ was achieved.

\section{Conclusions}

The assessment of the LOS characteristic of ITER Bolometer prototype collimators has demonstrated its usefulness by identifying different construction problems. Different characteristics like reflections on the side walls and the microwave grid diffraction could be identified. IBOROB showed that it can help the evolutionary prototyping process of the ITER bolometers. It provides enough information so that the collimators can be continually improved. The collimator design can be guided by combining and varying results of different measurement series. The final design will presumably be a combination of multiple design versions.

All characteristics in the measured transmission functions could not be identified but the research in this area continues. Other design development steps will be elaborated, and further variations of aperture designs need to be considered. One potential development step is to reduce the aperture thickness because there seem to be reflections inside the aperture itself.

The facility IBOROB is also a demonstration technology defining the main parameters of a pathway for how such measurements could be performed on other diagnostics for fusion devices. Currently IBOROB is under development to be used as a calibration device for the in situ measurement of the LOS of the bolometers in ASDEX Upgrade. The experience gained during this analysis will help to provide the basis for defining one for ITER, compatible to the remote handling tools foreseen in [4].

Developments are ongoing to determine the complete two-dimensional viewing cone of each LOS. However to accelerate the measurement procedure and to make binding statements considering the absolute orientation, the external calibration with the FARO measurement arm has to be implemented in the automation procedure in LabVIEW.

\section{Acknowledgments}

The authors wish to explicitly thank the students D. H. Nguyen and T. N. Lee who helped to perform and develop further the laboratory measurements with IBOROB.

This work was supported by funds from the German Ministry for Education and Research under the Grant No. 03FUS0006. The sole responsibility for the content presented lies with the authors.

\section{References}

[1] H. Meister, F. Penzel, L. Giannone, M. Kannamüller, A. Kling, J. Koll, T. Trautmann, Development of an automated method for in situ measurement of the geometrical properties of the ITER bolometer diagnostic, Fusion Engineering and Design 86 (68) (2011) 1170 - 1173, proceedings of the 26th Symposium of Fusion Technology (SOFT-26)

[2] L. C. Ingesson, C. F. Maggi, R. Reichle, Characterization of geometrical detection-system properties for two-dimensional tomography, Review of Scientific Instruments 71 (3) (2000) 1370-1378.

[3] L. Giannone, K. Mast, M. Schubert, Derivation of bolometer equations relevant to operation in fusion experiments, Review of Scientific Instruments 73 (9) (2002) 3205 -3214.

[4] I. Ribeiro, C. Damiani, A. Tesini, S. Kakudate, M. Siuko, C. Neri, The remote handling systems for ITER, Fusion Engineering and Design 86 (6-8) (2011) 471-477. 